\title{
Foot ulcers and risk factors among diabetic patients visiting Surgery Department in a University Teaching Hospital in Ajman, UAE
}

\author{
Venkatramana Manda', Jayadevan Sreedharan², Jayakumary Muttappallymyalil ${ }^{2}$, Rajdeep Das ${ }^{3}$ \\ and Emi Hisamatsu ${ }^{4}$
}

\author{
${ }^{1}$ Department of Surgery, College of Medicine, Gulf Medical University, Ajman, UAE \\ ${ }^{2}$ Research Division, Gulf Medical University, Ajman, UAE \\ ${ }^{3}$ Department of Pathology, Gulf Medical University, Ajman, UAE \\ ${ }^{4}$ University of Auckland, New Zealand
}

\section{A B STRACT}

\begin{abstract}
Objective: To assess the frequency of foot ulcers and their risk factors among diabetic patients visiting surgery department in a university teaching hospital in Ajman, UAE especially in relation to gender, nationality and age, blood glucose levels and lipid profile. Materials and Methods: This hospital-based retrospective record analysis was conducted for patients with diabetes mellitus visiting department of surgery for a period of 8 years from 2002 to 2010. Diabetic patients with foot ulcers were cases and those without were controls. Results: Among the diabetic patients recruited for this study, $75 \%$ were males and $25 \%$ were females. Among these diabetic patients, $76.3 \%$ of male patients were diagnosed with foot ulcers as opposed to $23.8 \%$ of female patients. Majority of diabetic patients were from Asia (53.1\%) and MiddleEast $(33.8 \%)$. Diabetic foot ulcers were reported more in Asian patients (57\%) compared to patients from Middle East (29.1\%). Diabetic foot ulcers were most prevalent in the age group 50 to 59 years $(42.5 \%)$ and least prevalent in the age group 20 to 29 years (5\%). The mean HDL, total cholesterol, LDL and VLDL were all higher for diabetic patients with foot ulcer than controls with levels of only HDL being statistically significant $(p<0.05)$. Mean HDL levels for cases was $37.3 \pm 8.3$ whereas that for controls was $31.3 \pm 7.9$. The mean Post Prandial blood glucose is $286.4 \mathrm{mg} / \mathrm{dl}$ for diabetic patients without foot ulcer which is significantly higher than diabetic patients with foot ulcer who had a mean value of $249.6 \mathrm{mg} / \mathrm{dl}$ but there was no significant difference in mean fasting glucose values. Conclusion: Asian middle-aged male diabetics are most likely to have foot complications. HDL levels were high whereas post-prandial blood glucose levels were comparatively low in diabetics with foot ulcers than without.
\end{abstract}

Keywords: Middle-east, diabetic foot ulcer, diabetic neuropathy, peripheral arterial disease, amputations, dyslipidemia, risk factors

\section{INTRODUCTION}

Diabetes Mellitus (DM) is one of the most widespread non communicable diseases across the world. Two types of complications are encountered usually with DM: microvascular and macrovascular. Diabetic neuropathy

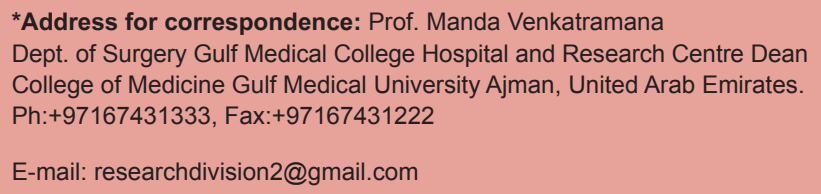

DOI: $10.5530 /$ ijmedph.2.3.8 is one of the most commonly-occurring microvascular complications, of which the most common type is distal symmetrical neuropathy or polyneuropathy. Patients who present with diabetic foot ulceration are heterogeneous. Even though most of them have peripheral polyneuropathy, there are several other factors that may vary among patients, such as the existence of peripheral arterial disease (PAD), infection and co-morbid conditions. PAD is present in approximately one-half of patients with foot ulcers. ${ }^{1}$ Diabetic foot ulcers continue to be a major cause of morbidity and results in an amputation rate of $19 \%$ in a hospital based one year study in Saudi Arabia ${ }^{2}$ and in other the Arab world, the incidence is still higher. ${ }^{3-5}$ 
Diabetes-related conditions in the lower limb that increases the risk for amputation among people having DM include peripheral neuropathy, PAD, and sepsis. Peripheral neuropathy cause loss of sensation, resulting in inability to recognize foot problems and may lead to development of foot deformities that increase pressure points prone to ulceration. Common risk factors for amputation include older age, male gender, a member of certain racial/ethnic groups, having poor glucose control, having diabetes for a long duration and practicing poor preventive health care. ${ }^{6}$ It is estimated that up to $15 \%$ of all people with diabetes will eventually develop a foot ulcer and up to $10 \%$ of all non-traumatic amputations are performed on patients with diabetes. ${ }^{7}$ Singh et $\mathrm{al}^{8}$ in their study report that the prevalence of foot ulcers is $4 \%$ to $10 \%$, the annual population-based incidence is $1.0 \%$ to $4.1 \%$ and the lifetime incidence may be as high as $25 \%$.

The ENDCAD study (The Emirates National Diabetes Study and Screening for Coronary Artery Diseases Risk Factors), jointly conducted by the $\mathrm{WHO}$ and the United Arab Emirates (UAE) Ministry of Health (1998-2000) among those above 21 years of age, showed a prevalence of DM as $19.6 \%$ and $15.2 \%$ of Impaired glucose tolerance (IGT). Among the UAE nationals, it was $24 \%$ DM and $17.9 \%$ IGT and among expatriates $17.4 \% \mathrm{DM}$ and $13.4 \%$ of IGT. According to this report, UAE rank second highest in the world and the number is increasing dramatically. ${ }^{9}$ Though foot ulcers and amputation are common complications of diabetes mellitus, foot ulceration and amputation does not have to be an expected outcome of diabetes. Improving glucose control and educating patients in the routine care of their feet can reduce the incidence of diabetic foot complications. The frequency of foot ulcers and their risk factors among diabetic patients visiting surgery department in a university teaching hospital in Ajman, UAE especially in relation to gender, nationality and age, blood glucose levels and lipid profile was assessed in the present study.

\section{MATERIALS AND METHODS}

This hospital-based retrospective record analysis was conducted for patients with diabetes mellitus with or without foot ulcers visiting department of surgery for a period of 8 years, from 2002 to 2010. GMU Ethics and Research Committees approval was sought before starting the research. Proper confidentiality of patient's identification was taken under consideration. Patient's name, telephone number and other identification which may jeopardize the issue of confidentiality were not included.

All diabetic patients (both Type I and II) who had visited surgery department GMC Hospital and Research Center in the period from 2002 to 2010 formed the study population. All diabetic patients (both Type I and II) with diabetic foot or diabetes-related foot ulcers were considered as cases and all diabetic patients without diabetic foot or diabetes-related ulcers were considered as controls, irrespective of their complications. Patients other than diabetes irrespective of presence or absence of non-healing leg ulcers were excluded from this study. A checklist was prepared to enter the data already recorded in the case record files. This checklist was pilot-tested by recording data from five case records before finalizing the checklist. A Research Assistant was involved in collection of data from the Medical Records Department after obtaining permission from the Chief of Medical Records. Predictive Analytic Software version 18 was used for data analysis. Descriptive statistics and student t test was performed to compare the mean values in diabetics with and without foot ulcers.

\section{RESULTS}

For this study, 52 patients with DM only and 80 patients with DM foot ulcers were included. With regard to gender distribution, among cases and controls, males were more compared to females. Among cases $76.3 \%$ and among controls $73.1 \%$ were males. While considering the distribution of ethnicity, more than $80 \%$ of cases and controls were from Middle East or from Asian regions. Age distribution showed more than $75 \%$ of the cases and controls were in the age group of 30-60 years. The details of the distribution of demographic variables are given in Table 1.

In some of the cases and control subjects, the lipid profile values were not available and hence the comparison of lipid profile was done with available data. The mean values of lipid profile was high among cases compared to controls but was not statistically significant except HDL $(p<0.05)$. The mean HDL value for diabetic patients with foot ulcers was $37.3 \pm 8.25$ whereas that for the non-foot ulcer patients is $31.3 \pm 7.85$. The details about the lipid profile is given in Table 2.

Furthermore, the mean value of post prandial blood glucose is significantly higher for the diabetic patients without 
Table 1: Demographic variables among DM patients with and without foot ulcer

\begin{tabular}{|c|c|c|c|c|c|}
\hline \multirow[t]{2}{*}{ Variables } & \multirow[t]{2}{*}{ Group } & \multicolumn{2}{|c|}{ DM only } & \multicolumn{2}{|c|}{ DM with Foot ulcer } \\
\hline & & No & $\%$ & No & $\%$ \\
\hline \multirow[t]{2}{*}{ Gender } & Female & 14 & 26.9 & 19 & 23.8 \\
\hline & Male & 38 & 73.1 & 61 & 76.3 \\
\hline \multirow[t]{4}{*}{ Ethnicity } & Middle East & 21 & 41.2 & 23 & 29.1 \\
\hline & Asia & 24 & 47.1 & 45 & 57.0 \\
\hline & Africa & 5 & 9.8 & 11 & 13.9 \\
\hline & Europe & 1 & 2.0 & - & - \\
\hline \multirow[t]{6}{*}{ Age } & $20-29$ & 3 & 5.8 & 4 & 5.0 \\
\hline & $30-39$ & 14 & 26.9 & 13 & 16.3 \\
\hline & $40-49$ & 11 & 21.2 & 17 & 21.3 \\
\hline & $50-59$ & 14 & 26.9 & 34 & 42.5 \\
\hline & $60-69$ & 8 & 15.4 & 7 & 8.8 \\
\hline & $70-79$ & 2 & 3.8 & 5 & 6.3 \\
\hline Total & & 52 & - & 80 & - \\
\hline
\end{tabular}

\begin{tabular}{|c|c|c|c|c|c|}
\hline Parameters & Group & $\mathbf{N}$ & Mean & SD & $\mathbf{P}$ \\
\hline Total & Control & 32 & 175.75 & 56.49 & NS \\
\hline Cholesterol (mg/dL) & Cases & 18 & 189.89 & 66.79 & \\
\hline \multirow[t]{2}{*}{$\mathrm{HDL}(\mathrm{mg} / \mathrm{dL})$} & Control & 32 & 31.34 & 7.85 & $<0.05$ \\
\hline & Cases & 18 & 37.28 & 8.25 & \\
\hline \multirow[t]{2}{*}{ LDL (mg/dL) } & Control & 30 & 102.07 & 39.61 & NS \\
\hline & Cases & 18 & 103.33 & 39.31 & \\
\hline \multirow[t]{2}{*}{ VLDL (mg/dL) } & Control & 29 & 35.46 & 16.31 & NS \\
\hline & Cases & 18 & 48.17 & 41.29 & \\
\hline \multirow[t]{2}{*}{ TG (mg/dL) } & Control & 32 & 207.38 & 122.24 & NS \\
\hline & Cases & 19 & 186.37 & 149.30 & \\
\hline
\end{tabular}

foot ulcers with a mean of 286.4 as compared to 249.6 for the diabetic patients with foot ulcers. There was no statistically significant difference in the fasting blood glucose level of cases and controls, but the mean value was high among cases compared to controls. The details are given in Table 3.

\section{DISCUSSION}

Foot ulcers among diabetics are a major cause of mortality and morbidity and also a huge financial burden on health care services as inadequate and improper treatment could result in development of gangrene leading to limb amputations and sometimes even death. The significance lies in the fact that identification of risk factors is important to screen high-risk patients to prevent development of foot ulcers and its associated morbidity. ${ }^{10}$ The greatest risk factors for developing foot ulceration are neuropathy, arterial disease, foot deformity and peripheral neuropathy accounting for $80 \%$ of diabetic persons with foot ulcers. The contributing factors are poor glycemic control and microvascular disease. ${ }^{11}$ Socio-demographic factors seem to play an important role in the development of foot ulcers in diabetics. Men are predominantly afflicted by foot ulcerations but studies have not been able to establish gender as an independent risk factor for developing foot ulcerations in diabetic patients. ${ }^{12}$ Our study also revealed a male preponderance.

In a retrospective study in a primary care setting observed that the mean age of being diagnosed having diabetes was $52 \pm 10.7$ years and mean age of being diagnosed having diabetic foot ulcer was $54.7 \pm 10.2$ years. ${ }^{13}$ The findings are

\begin{tabular}{|c|c|c|c|c|c|}
\hline Blood Glucose (gm/L) & Group & $\mathbf{N}$ & Mean & SD & $p$ value \\
\hline \multirow[t]{2}{*}{ Fasting } & Control & 37 & 179.49 & 57.691 & NS \\
\hline & Case & 76 & 181.55 & 63.116 & \\
\hline \multirow[t]{2}{*}{ Post Prandial } & Control & 43 & 286.40 & 86.747 & $<0.05$ \\
\hline & Case & 76 & 249.61 & 45.560 & \\
\hline
\end{tabular}


similar to our study with the greatest incidence of diabetics with foot ulcer being between 50-59 years of age.

In a study conducted on 60 diabetic patients, 30 with foot ulceration and 30 without foot lesions, the mean $\mathrm{HbA1c}$ level in diabetics without ulcers was $9.77 \pm 2.34$, which was significantly lower than the corresponding level in diabetics with ulcers was $14.14 \pm 3.63$ suggesting that foot ulcers are more likely to occur in poorly controlled diabetic patients ${ }^{14}$ this findings does not correlate with our study which showed that the mean fasting and post-prandial sugar levels were more in patients without foot ulcers than with foot ulcers.

In a descriptive observational study among 100 patients, the incidence of poor glycemic control was $69 \%$ and dyslipidemia was $61 \% .{ }^{15}$ In another large series consisting of 1121 patients in a primary care setting, it was found that renal profile and lipid profile could be used as a predictor to ulcer-free survival for diabetic foot ulcer. ${ }^{16}$ Our study did reveal a correlation between dyslipidemia and presence of foot ulcers. The mean values of all lipids except triglycerides were more for the diabetic patients with foot ulcer group than without but the only value which was statistically significant was HDL levels. This could be attributed to inability to adhere to an exercise regime due to presence of foot ulcers. The differences observed in levels of total cholesterol, LDL, VLDL and triglycerides between the two groups were not significant. The mean value for triglycerides does not follow the pattern like the other values but this may be due to sampling errors.

Though foot ulcers and amputation are common complications of diabetes mellitus, foot ulceration and amputation does not have to be an expected outcome of diabetes. Avoiding risk factors, such as inappropriate blood glucose control, could reduce the risk of neuropathy. Thus improving glucose control, proper foot care and education for the total population is of great importance for neuropathic patients to reduce the risk of foot ulceration and potential amputation. A multidisciplinary healthcare team including physicians, podiatrists, diabetes educators and tissue viability nurses is essential to provide a comprehensive approach to care of patients with diabetes.

\section{CONCLUSION}

Asian middle-aged male diabetics are most likely to have foot complications. It can be drawn from this study that socio-demographic factors like age, gender and ethnicity along with modifiable factors like glycemic control and dyslipidemia are associated with foot ulcers in diabetic patients. It is binding on the health care provider to control these modifiable risk factors to prevent development of complications in diabetic patients with foot ulcers and improve the quality of life.

\section{RECOMMENDATIONS}

It is important to educate all diabetic patients about the importance of self-examination of both feet and physicians should routinely examine the feet of all diabetic patients which would go a long way in preventing development of foot ulcers. With this high incidence of diabetic foot complications there is a need for launching the diabetic foot care program at Gulf Medical College Hospital and Research Centre, Ajman, UAE, which is a comprehensive approach to maintaining the health of diabetic patients' feet in order to reduce the lower limb amputation rate, thereby gradually reducing the economic burden to patients, community at large and the health care system.

\section{REFERENCES}

1. Prompers $\mathrm{L}$, Huijberts $\mathrm{M}$, Apelqvist $\mathrm{J}$, et al. High prevalence of ischaemia, infection and serious comorbidity in patients with diabetic foot disease in Europe. Baseline results from the Eurodiale Study. Diabetologia 2007;50:18-25.

2. Al-Tawfiq, Jaffar A, John Drow, Jane A. Presentation and Outcome of Diabetic Foot Ulcers in Saudi Arabian Patients. Advances in Skin \& Wound Care 2009;22(3):119-121. doi: 10.1097/01.ASW.0000305450.33693.f8.

3. Al-Wahbi AM. The diabetic foot in the Arab world. Saudi Med J 2006; 27:147-53.

4. Wrobel JS, Mayfield JA, Reiber GE. Geographic variation of lower extremity major amputation in individuals with and without diabetes in the Medicare population. Diabetes Care 2001;24:860-4.

5. Al-Wahbi AM. The diabetic foot in the Arab world. In: Gill G, editor. Diabetes in the Arab World. Cambridge: FSG Communications 2005.

6. Dargis V, Pantelejeva O, Jonushaite A, Vileikyte L, Boulton AJ. Benefits of a multidisciplinary approach in the management of recurrent diabetic foot ulceration in Lithuania: A prospective study. Diabetes Care 2009;22:1428-31.

7. Department of Health. Improving diabetes services: The NSF two years on. Department of Health, London. Available from: URL: www.dh.gov.uk/ PublicationsAndStatistics/Publications/Publications Policy And Guidance/ Publications Policy And Guidance Article/fs/ en. 2005. (Accessed 12th February 2011).

8. Singh N, Armstrong DG, Lipsky BA. Preventing foot ulcers in patients with diabetes. JAMA 2005;293:217-28.

9. National Diabetes Committee. National Diabetes Guidelines United Arab Emirates 2009. Available from: URL:http://www.dcc.org.ae/Uploads/file/ National\%20Diabetes\%20Guidelines\%20UAE.pdf. (Accessed on $30^{\text {th }}$ March 2011)

10. Thanh D, Aristidis V. The Influence of Gender as a Risk Factor in Diabetic Foot Ulceration. Available from: URL:http://www.woundsresearch.com/ article/8707. (Accessed on 10th July 2011).http://www.woundsresearch. com/article/8707. (Accessed on 10th July 2011). 
11. Vincent Lopez Rowe. Diabetic Ulcers. Available from: URL: http://emedicine. medscape.com/article/460282-overview. (Accessed on 10th July 2011).

12. Faridah K, Azmi MT. Retrospective study of predictors for foot ulceration among diabetic patients attending Kuala Langat health centre from 1999 to 2008. Journal of Community Health 2009:15(2): 43-59.

13. Ramani A, Nayak SS, Gopalakrishna K, Kundaje GN. Glycemic control and its relationship to diabetic foot ulcers; Indian J Pathol Microbiol 1991;34(3):161-5.
14. Hamza K, Zahidullah K, Inamullah K, Jamal ud Din, Sadiq U, Bughdad K. Factors contributing to the development of diabetic foot ulcers and role of health literacy. Available from; URL: http://www.scopemed.org/ mnstemps/27/27-1298387017.pdf. (Accessed on July 10th 2011).

15. Katy Dunn. Preventing amputation in patients with diabetes Wounds UK Clinical Practice Development 2007;3(1):22-30. 